\title{
Evaluación de la carga y fatiga mental en docentes por teletrabajo a causa del COVID-19
}

\section{Evaluation of the mental burden and fatigue in teachers by teleworking caused by covid-19}

\author{
Danilo Andrés Terán Villacís. ${ }^{1}$, Manolo Alexander Córdova Suárez. ${ }^{2}$, Juan Pablo
} Muquinche Puca. ${ }^{3} \&$ Paulina del Roció Gordón Villalba. ${ }^{4}$

Recibido: 10-10-2020 / Revisado: 15-11-2020 /Aceptado: 04-12-2020/ Publicado: 02-01-2021

Abstract.

DOI: $\quad$ https://doi.org/10.33262/cienciadigital.v5i1.1515

Introduction: A mental load study was carried out on 103 teachers from different universities in central Ecuador using the NASA-Task Load Index (LTX) method, in the study the mental, physical, time, effort, performance and the frustration level were quantified to different age groups, to detect the psychosocial risk factor and incidence in normal fatigue and chronic fatigue. Methods: A sample of 103 university teachers divided into 9 age segments and from different educational institutions in the central area of Ecuador that carry out teaching-learning activities in telework mode was used. First, a survey was applied according to the NASATask Load Index (LTX) method, to quantify the 6 factors or dimensions considered by the method. Then a health questionnaire related to simple and chronic mental load was applied. This research was carried out in two phases, the first for weighting and the second for scoring. Results: The application of the NASA LTX method managed to detect that the greatest affectation by mental load is in the age group of: 5559 years with a score of 1250, then those from 40 to 44 years with a value of 1020 and those from 50 to 54 years with a value of 1010 ,

\footnotetext{
${ }^{1}$ Regional Autonomous University of Los Andes, Postgraduate, Ambato, Ecuador, pg.daniloatv80@uniandes.edu.ec, ID de ORCID 0000-0001-8998-5026

${ }^{2}$ G+ Energy-Risks \& Engineering Group, Department of Food Science and Engineering, Technical University of Ambato, Ambato-Ecuador, ma.cordova@uta.edu.ec, ID de ORCID 0000-0001-6786-7926

${ }^{3}$ Abkrea Ingeniería Cía. Ltda., Ambato, Ecuador, jp.muquinche@ gmail.com ID de ORCID 0000-0002-5286-4076

${ }^{4}$ Faculty of Health Sciences, Technical University of Ambato, Ambato-Ecuador, pdr.gordon@uta.edu.ec ID de ORCID 0000-0002-1416-7560
} 
then follow the other groups with values less than 100. The mental fatigue value determines values of $100 \%$ of affectation to the group of 35 to 39 years of age and 50 to 54 years an $87.5 \%$ of affectation to the group of 40 to 44 years of age, the other groups present values less than $75 \%$ of affectation by discomfort of mental fatigue. All groups present simple and chronic fatigue complaints. Conclusion: The results identify as the group most affected by load and mental fatigue, teachers who are over 39 years of age due to telework activities. On the other hand, the affectation of simple and chronic mental fatigue is proportional and significantly affects all groups

Keywords: Telework, Mental load, Mental Fatigue, University teacher, Covid-19

\section{RESUMEN}

Introducción: Se realizó un estudio de carga mental a 103 docentes de distintas universidades del centro del Ecuador utilizando para esto el método NASA-Task Load Index (LTX), en el estudio se cuantificó la exigencia mental, física, temporal, esfuerzo, rendimiento y el nivel de frustración a diferentes grupos de edades, para detectar el factor de riesgo psicosocial e incidencia en la fatiga normal y fatiga crónica. Métodos: Se utilizó una muestra de 103 docentes universitarios divididos en 9 segmentos de edades y de distintas instituciones educativas de la zona centro del Ecuador que ejecutan actividades de enseñanza aprendizaje en modalidad de teletrabajo. Primero se les aplicó una encuesta según el método NASA-Task Load Index (LTX) para cuantificar los 6 factores o dimensiones que considera el método. Luego se aplicó un cuestionario de salud relacionado a la carga mental simple y crónica. Esta investigación se efectuó en dos fases, la primera de ponderación y la segunda de puntuación. Resultados: La aplicación del método NASA LTX logró detectar que la mayor afectación por carga mental está en el grupo de edad de: 5559 años con un puntaje de 1250, luego los de 40 a 44 años con un valor de 1020 y los de 50 a 54 años con un valor de 1010, luego siguen los demás grupos con valores menores de 100. El valor de fatiga mental determina valores del100\% de afectación al grupo de 35 a 39 años de edad y 50 a 54 años un 87,5 \% de afectación al grupo de 40 a 44 años de edad, los demás grupos presentan valores menos del $75 \%$ de afectación por molestias de fatiga mental. Todos los grupos presentan molestias de fatiga simple y crónica. Conclusión: Los resultados identifican como grupo más afectado por carga y fatiga mental a docentes que superan los 39 años de edad por actividades de teletrabajo. Por otro lado, la afectación por fatiga mental simple y crónica es proporcional y afecta significativamente a todos los grupos

Palabras Claves: Teletrabajo, Carga mental, Fatiga Mental, Docente universitario, Covid-19 Introducción 
Para controlar el impacto de la pandemia causada por Covid-19 en todo el mundo se han aplicado restricciones a todo nivel (Wang et al., 2021)y especialmente en a las actividades que se pueden ejecutar como un trabajo en casa (Mheidly et al., 2020). El trabajo se concentró en un 34\% a teletrabajo (Peiró y Soler, 2020) y a porcentajes cerca del 59,9\% en actividades académicas e intelectuales. El 16 de marzo 2020, mediante Decreto Ejecutivo 1017, el Gobierno Nacional del Ecuador declara el estado de excepción por calamidad pública en todo el territorio nacional disponiendo entre otras medidas la suspensión de las libertades de tránsito, movilidad humana y sobre todo clases virtuales a todo nivel educativo empezando una nueva forma de trabajo y de adaptación de los usuarios y docentes que ejecutan estas tareas. Estas nuevas maneras de enseñanza aprendizaje y la falta de preparación de los involucrados en muchos casos se cree que son factores determinantes en los problemas de rendimiento, eficiencia pero especialmente en el aumento de los factores de riesgo psicosocial (Venegas Tresierra y Leyva Pozo, 2020).

Este aislamiento dentro de la estructura familiar generó cambios importantes por la interacción cercana entre los miembros de las familias (Santillán-Marroquín, 2020) y sobre todo el uso de herramientas informáticas y manejo de mucha información para justificar tareas de los estudiantes y trabajo de los docentes. Este cambio radical del modelo educativo no tiene precedentes históricos en el mundo pues es una situación que surge de la crisis sanitaria causada por una pandemia que agobia al mundo entero y la educación ha tenido que reinventarse para dar continuidad a los procesos de formación de carreras universitarias y ha generado nuevas exigencias de desempeño además de la improvisación de estrategias de enseñanzas completamente diferentes a las presenciales, dedicación de tiempos extraordinarios para la investigación y preparación de materiales en plataformas informáticas (Martínez-Garcés y Garcés-Fuenmayor, 2020) educativas promovidos por los directivos de las universidades y con la participación de estudiantes poco receptivos y menos dispuestos a permanecer frente al computador durante horas (Cueva y Terrones, 2020), en un modelo tan distinto de formación; está provocando reacciones físicas, psicológicas y emocionales que afectan al docente universitario que pueden derivar en carga y fatiga mental excesiva en tal medida que sobrepasen la capacidad de adaptación y produzcan resultados desastrosos no solamente en su desempeño sino además en su calidad de vida (Vivanco-Saraguro, 2020).

La importancia de establecer una línea base para detección y atención de factores psicosociales que afectan al sector laboral de la docencia resulta prioritario ya que no es precisamente un aspecto que preocupe al nivel directivo de las instituciones de educación superior y aunque las exigencias en seguridad y salud ocupacional obliga a la detección, identificación, prevención y adopción de medidas para garantizar la salud y neutralizar los riesgos ocupacionales, en la práctica no surgen acciones concretas para abordar y tratar los factores de riesgo psicosocial que afectan a los docentes del sistema de educación superior y menos 
aún se sabe que grupo es el más sensible y necesita actuación inmediata (Martínez Barranco y Yandún Burbano, 2017). Este trabajo intenta abordar esta problemática y ayudar a establecer las pautas para establecer un programa de riesgo psicosocial adecuado y efectivo.

En esta investigación nuestro objetivo fue evaluar el impacto de la epidemia de COVID-19 en la carga de trabajo y la salud mental del personal docente universitario de la zona sierra centro del Ecuador mediante el Cuestionario de índice de carga de tareas de la NASA-Task Load Index (LTX), en un periodo de seis meses entre marzo y septiembre de 2020. Aunque existe un gran número de métodos de evaluación de riesgos psicosociales son diversos y dependen de los elementos específicos a evaluarse (Hinojosa et al., 2019), la mayoría de instrumentos de evaluación de carga mental considera seis dimensiones definidas es por ello que el método NASA-Task Load Index (LTX), resulta efectivo y de rápida aplicación.

\section{Materiales y Método}

\section{Determinación de la muestra}

Se utilizó un muestreo estratificado para separar la población en segmentos exclusivos (Kish, 1972). Para este caso la muestra está compuesta por 103 docentes universitarios, 30 hombres y 73 mujeres de distintas universidades del país, durante el periodo 2020.

\section{Evaluación de la carga mental}

Los desencadenantes de la carga mental en ambientes laborales son variados y de diversa naturaleza sin embargo la carga mental se presenta como el factor de riesgos relacionado a la tarea más susceptible de provocar estrés laboral y sobre todo con las tareas que imponen máxima concentración y atención a los detalles (Valdehita et al., 2007).

Múltiples son los factores que la determinan pero los que se han estudiado y son de relevancia conlleva la interacción de: el ambiente físico de trabajo, las relaciones interpersonales, las características personales, la duración de la tarea, la complejidad del trabajo, la implicación emocional en el modelo de tareas que cumple en soledad (Valdehita et al., 2001).

Esta investigación utiliza uno de los métodos cualitativos y cuantitativos que la literatura especializada considera como los más idóneos (Yuliani y Tambunan, 2018). Hay que anotar que de todas las herramientas y métodos cualitativos los más utilizados por sus cuestionarios son: el inventario sueco de fatiga ocupacional (SOFI) (López et al., 2018) y el método NASATask Load Index (LTX) y entre los métodos cuantitativos que son más aceptados está el que consideran el uso de la frecuencia cardíaca y gasto energético. 
Para este trabajo se utilizó el método NASA-Task Load Index (LTX) ya que determina una aplicabilidad directa y una ponderación numérica final para mejor entendimiento (Alvarado Bautista y Sánchez Merchán, 2020), el cual considera seis dimensiones como principales: las exigencias mentales, físicas, temporales, se considera el factor de rendimiento, el grado de esfuerzo y también el nivel de frustración durante la ejecución de las actividades o tareas con exposición.

El método NASA-Task Load Index (LTX) se aplica en dos fases bien definidas. Se empieza con preguntar la importancia que manifiesta el objeto de análisis de las 6 dimensiones y sus 15 comparaciones de pares para tener un valor ponderado como primera aproximación de la opción que considera de mayor importancia. Finalmente se utiliza una plantilla de preguntas que el individuo escoge como la realidad de su situación de cada una de los factores de carga mental de una escala establecida de 0 a 100 (de Arquer y Nogareda, 2000).

\section{Fatiga Mental}

Este trabajo toma en cuenta los efectos de la fatiga simple y crónica en un cuestionario de aplicación directa (Barbado Hernández et al., 2006) con preguntas de si o no sobre la afectación en cuanto:

a. bajo rendimiento,

b. síntomas de cansancio,

c. pérdida de atención en la ejecución de actividades,

d. sentimiento de irritabilidad,

e. síntomas de ansiedad,

f. síntomas de insomnio,

g. síntomas de mareo. No se consideró índice de morbilidad por la ausencia de los dispensarios de salud ocupacional en tiempo de pandemia.

\section{RESULTADOS}

\section{Resultado carga y fatiga mental en docentes.}

La aplicación del método se ejecutó en el final del primer semestre de pandemia y se observa los siguientes resultados. Ver gráfico 1 


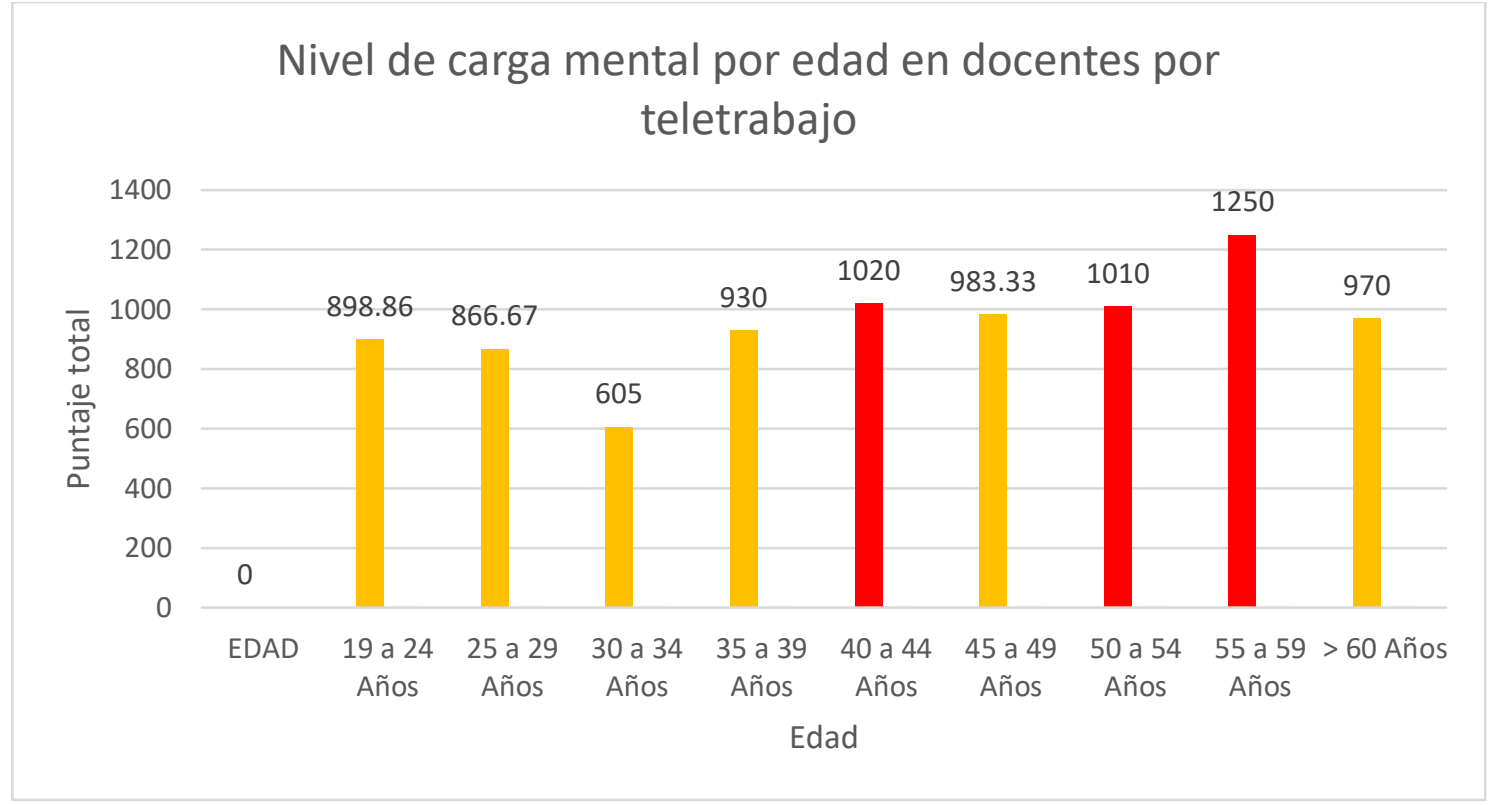

Figura 1. Establece los valores del nivel de carga mental como resultado de la aplicación del método NASA LTX por grupo de edad.

Al aplicar el cuestionario de fatiga mental se observan los siguientes resultados.

Ver Figura 2.

\section{$\%$}

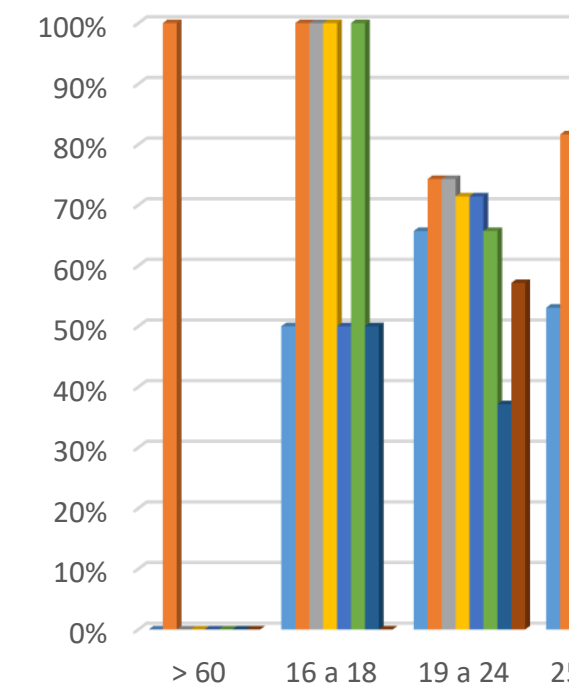

\section{Fatiga mental en docentes universitarios por teletrabajo}

Figura 2. Establece los valores de fatiga mental por pregunta.— P1: bajo rendimiento,_ P2: síntomas de cansancio, — P3 pérdida de atención, —P4 síntomas de irritabilidad, — P5 síntomas de ansiedad, P6 insomnio, $\square$ P7 Mareos, $\square$ P8 mal diseño de puesto. 


\section{Discusión}

Las características demográficas de la muestra de profesores universitarios que aceptaron participar en el estudio fueron las siguientes: de un total de 103 participantes, un $70,1 \%$ fueron mujeres, el 29,9\% hombres, un 34,65\% fueron docentes de entre 19 a 24 años, el 48, 51\% tenían entre 25 a 29 años, $0,99 \%$ de 30 a 34 años, $0,99 \%$ de 35 a 39 años, un 7,92 \% edades entre los 40 a 44 años, 0,99\% 45 a 49 años, 0,99\% 50 a 54 años, 1,98\% de entre 55 a 59 años y un $0,99 \%$ edades superiores a 60 años, en relación a la preparación académica, el 100\% tenía título de tercer grado o superior.

La investigación concluye que el nivel de carga mental promedio en el grupo estudiado y evaluado de acuerdo al método NASA LTX es de 948 lo que según ese método de análisis es un valor medio aunque muy cercano al valor inferior de alto (1000), también se detectó que los grupos más afectados son aquellos comprendidos en edades de 39 años y superiores. Estos resultados determinan valores de carga mental sobre 500 situación similar a los datos publicados por Hinojosa en el 2019 pero por situaciones de profesores universitarios impagos (Hinojosa et al., 2019) aunque no determina afectación por edades.

\section{Conclusiones}

- La aplicación del método NASA LTX logró detectar que la mayor afectación por carga mental está en el grupo de edad de: 5559 años con un puntaje de 1250, luego los de 40 a 44 años con un valor de 1020 y los de 50 a 54 años con un valor de 1010, luego siguen los demás grupos con valores menores de 100.

- El valor de fatiga mental determina valores del100\% de afectación al grupo de 35 a 39 años de edad y 50 a 54 años un 87,5 \% de afectación al grupo de 40 a 44 años de edad, los demás grupos presentan valores menos del $75 \%$ de afectación por molestias de fatiga mental. Todos los grupos presentan molestias de fatiga simple y crónica.

- Los resultados identifican como grupo más afectado por carga y fatiga mental a docentes que superan los 39 años de edad por actividades de teletrabajo. Por otro lado, la afectación por fatiga mental simple y crónica es proporcional y afecta significativamente a todos los grupos

\section{Referencias Bibliográficas}

Alvarado Bautista, Á. M. y Sánchez Merchán, L. F. (2020). Carga mental del trabajo de personal (Psiquiatras) que labora en una institución hospitalaria en Bogotá a través del método NASATLX. 1, 2.

Barbado Hernández, F., Gómez Cerezo, J., López Rodríguez, M. y Vázquez Rodríguez, J. (2006). El síndrome de fatiga crónica y su diagnóstico en Medicina Interna. Paper presented at the Anales de medicina interna. 
Cueva, M. A. L. y Terrones, S. A. C. (2020). Repercusiones de las clases virtuales en los estudiantes universitarios en el contexto de la cuarentena por COVID-19: El caso de la PUCP. Propósitos y Representaciones, 8(SPE3), 588.

de Arquer, I. y Nogareda, C. (2000). NTP 544: Estimación de la carga mental de trabajo: el método NASA TLX. Madrid: Instituto Nacional de Higiene y Seguridad en el Trabajo, Ministerio de Trabajo y Seguridad Social del Gobierno de Espa na.

Hinojosa, T. A. R., Suárez, M. A. C., Villalba, P. d. R. G., Pérez, J. G. V., Robalino, O. E. R. y Almeida, E. L. S. (2019). Evaluación de la carga y fatiga mental en docentes. caso de la Universidad Técnica de Ambato. Ciencia Digital, 3(2), 252-263.

Kish, L. (1972). Muestreo de encuestas: Trillas México.

López, J. M. A. M., Muñoz, E. L. G. y Baltazar, R. G. (2018). Fatiga laboral, mediante la aplicación del SOFI-SM en bomberos mexicanos. Revista Colombiana de Salud Ocupacional, 8(1).

Martínez-Garcés, J. y Garcés-Fuenmayor, J. (2020). Competencias digitales docentes y el reto de la educación virtual derivado de la covid-19. Educación y Humanismo, 22(39), 1-16.

Martínez Barranco, M. P. y Yandún Burbano, E. D. (2017). Seguridad y salud ocupacional en Ecuador: Contribución normativa a la responsabilidad social organizacional.

Mheidly, N., Fares, M. Y. y Fares, J. (2020). Coping With Stress and Burnout Associated With Telecommunication and Online Learning. Frontiers in Public Health, 8. doi: 10.3389/fpubh.2020.574969

Peiró, J. y Soler, A. (2020). El impulso al teletrabajo durante el COVID-19 y los retos que plantea. En COVID-19: IVIEExpress.

Santillán-Marroquín, W. (2020). El teletrabajo en el COVID-19. CienciAmérica, 9(2), 65-76.

Valdehita, S. R., Moreno, L. L., García, J. M. y Díaz, J. J. (2007). Modelos y procedimientos de evaluación de la carga mental de trabajo. EduPsykhé: Revista de psicología y psicopedagogía, 6(1), 85-108.

Valdehita, S. R., Ramiro, E. M. D. y García, J. M. (2001). Aspectos metodológicos de la evaluación subjetiva de la carga mental de trabajo. Archivos de prevención de riesgos laborales, 4(4), 160-168.

Venegas Tresierra, C. E. y Leyva Pozo, A. C. (2020). Fatigue and mental workload among workers: about social distancing. Revista española de salud pública, 94.

Vivanco-Saraguro, A. (2020). Teleducación en tiempos de COVID-19: brechas de desigualdad. CienciAmérica, 9(2), 166-175.

Wang, H., Miao, Q., Shen, L., Yang, Q., Wu, Y., Wei, H., Yin, Y., Zhao, T., Zhu, B. y Lu, W. (2021). Characterization of the aerosol chemical composition during the COVID-19 lockdown period in Suzhou in the Yangtze River Delta, China. Journal of Environmental Sciences (China), 102, 110-122. doi: 10.1016/j.jes.2020.09.019

Yuliani, E. y Tambunan, E. (2018). Reduce workload of security officers using NASA-LTX, SOFI, Heart Rate and Energy Expenditure Method. $M S \& E$, 453(1), 012065.

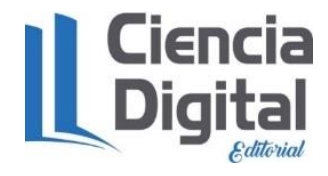


PARA CITAR EL ARTÍCULO INDEXADO.

Terán Villacís, D. A., Córdova Suárez, M. A., Muquinche Puca, J. P., \& Gordón Villalba, P. del R. (2021). Evaluación de la carga y fatiga mental en docentes por teletrabajo a causa del COVID-19 . Ciencia Digital, 5(1), 6-14. https://doi.org/10.33262/cienciadigital.v5i1.1515

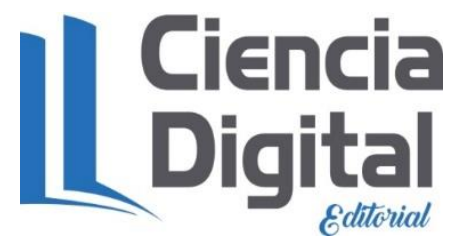

El artículo que se publica es de exclusiva responsabilidad de los autores y no necesariamente reflejan el pensamiento de la Revista Ciencia Digital.

El articulo queda en propiedad de la revista y, por tanto, su publicación parcial y/o total en otro medio tiene que ser autorizado por el director de la Revista Ciencia Digital.
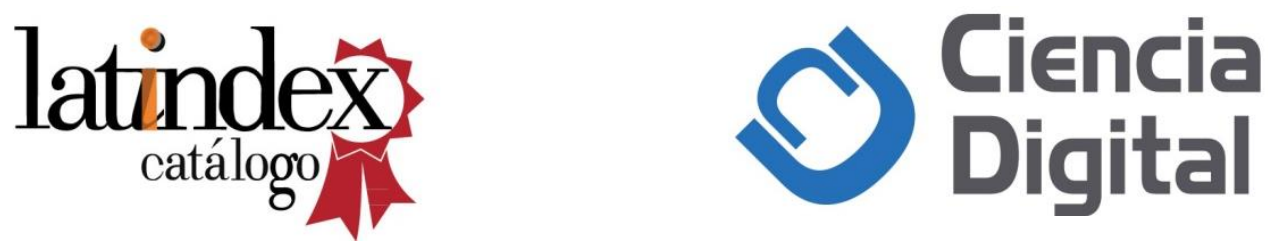